\title{
Pathways to success: AlME's educational mentoring model
}

\begin{tabular}{c|c}
$\begin{array}{c}\text { Amy Priestly } \\
\text { Australian Indigenous Mentoring Experience } \\
\text { (AIME) } \\
\text { ap@aimementoring.com }\end{array}$ & $\begin{array}{c}\text { Malcolm Lynch } \\
\text { Australian Indigenous Mentoring Experience } \\
\text { (AIME) }\end{array}$ \\
\hline $\begin{array}{c}\text { Carly Wallace } \\
\text { ml@aimementoring.com }\end{array}$ \\
$\begin{array}{c}\text { Australian Indigenous Mentoring Experience } \\
\text { (AIME) }\end{array}$ \\
cw@aimementoring.com \\
Professor Valerie Harwood \\
\hline
\end{tabular}

Keywords: Indigenous education, post-school transitions, AIME, storytelling, identity, support, mentoring.

\section{Introduction}

The Australian Indigenous Mentoring Experience (AIME) is a structured educational mentoring program provided for Indigenous students to access throughout their high school experience. The program is designed to support students to complete high school and transition into university, further education and training or employment at the same rate as every Australian child, effectively closing the gap on educational outcomes.

To better understand the impact of the program, AIME has developed a research partnership with a team of researchers from the University of Wollongong (UOW) and the University of Technology, Sydney (UTS). Comprising researchers with experience in qualitative and quantitative approaches, this research has grown from a small UOW internally funded project, to a large national project. This collaborative research partnership has already spanned five years and has produced several theorised academic papers, based on both qualitative and quantitative research that describes the AIME program and its merits (Bodkin-Andrews et al. 2013; O'Shea et al. 2013; Kervin et al. 2014; O'Shea et al. 2014; Harwood et al. 2015). Findings from the research to date have reported the success of the AIME program in engaging Indigenous young people in education (Bodkin-Andrews et al. 2013) as well as described the novel ways that the AIME program reorients the focus on aspirations to one of 'recognition of aspirations' (Harwood et al. 2014). Further analysis continues to be conducted, including the outcomes of an AIME national survey in 2014 and a paper currently under review that explains the different pedagogy used in the AIME program (McMahon et al, under review). All research activities through this partnership are aligned with protocols for research with Indigenous Australians, as described by the Australian Institute of Aboriginal and Torres Strait Islander Studies. We work collaboratively to discuss, plan and develop research projects and we have adapted data analyses approaches so we can engage in-group analysis so that a range of perspectives and epistemologies are able to be applied the collected data.

For this article, members of the AIME team decided that rather than repeating output from the current body of research, we would take a different approach and use the research as a jumping off point and provide a practitioners point of view and a perspective from the 'coal face' so we can share some of what we have found works within our program. Building on the research that 
has been published as well some of our more recent findings (currently being developed for publication), the AIME team sought to discuss the wide range of findings and subject them to further analysis. The authors yarned about what has been learned, what the research is saying and sought to understand more about how the program is working.

In this paper we describe three ways that AIME engages Indigenous young people, adding a richer and deeper narrative to the work that has been written about AIME to date. In this sense, the paper could be described as yarning about research and how we as AIME staff members yarned about and came to understand more deeply how the AIME program is engaging the young people. The paper is structured into two main sections. An overview of the AIME Program, its growth and its impact to date, followed by a section describing three of the key ways AIME educates and engages its students which in turn, gives a us a greater platform to transition AIME mentees into positive post school pathways.

\section{The AIME Program}

The AIME program is designed to support students to complete high school and transition into university, further education and training or employment at the same rate as every Australian child, effectively closing the gap on educational outcomes. Although there have been some recent improvements in closing the gap on educational outcomes between Indigenous and non-Indigenous students (SCRGSP, 2014), national statistics still show a significant difference. Grade progression, Year 12 attainment and post school transitions rates are all still considerably lower compared to non-Indigenous students and Indigenous Australians are underrepresented in the university sector, accounting for only $1.1 \%$ of all Australian university enrolments (Commonwealth of Australia, 2014). While it is reported that Indigenous young people are increasingly interested in attending university, enrolment and retention levels fall far below the levels required for equitable representation. The AIME program is addressing this inequality and the students involved are achieving great success. In 2014, AIME's junior high school students progressed through school on parity with non-Indigenous students, AIME's senior high school students were close to parity and the Year 12 cohort achieved results better than the national non-Indigenous rates (AIME, 2014). AIME students are also leading the way in closing the gap on post-school pathways with $76 \%$ of the AIME Year 12 cohort from 2014 successfully transitioning into university, further education and training or employment. And this isn't an overnight sensation; it has been building for some time. As the program has scaled from 325 mentees in 2009 to more than 4300 so far in 2015, AIME students have continued to achieve strong results and are entering university, further education, training and employment at record numbers. AIME's mentoring model deviates from the more traditional mentoring programs in a variety of ways. AIME was founded by young people, grew from the grass roots level and is Indigenous led. When AIME commenced, there was hardly any available research providing best practice for mentoring Australian Indigenous high school students. There was however there was a need to close the educational gap. To combat this, AIME is committed to building a body of research that continues to investigate and evaluate the impact of the program as well adding to the literature that helps to improve outcomes for Indigenous youth.

\section{Delivery modes}

The AIME program has three delivery modes. The AIME Institute delivered on campus at AIME's partner universities; Tutor Squads which are deployed into schools; and our one-onone coaching, career support and post-school transition support. 


\section{The AIME Institute}

The AIME Institute is run at universities campuses across Australia and offers six different courses tailored for each specific high school year group (Year 7-12) featuring 49 unique modules, each one hour in length. Many of the modules provide launch pads for real life opportunities for the students to extend themselves through, for example, internships for artists, performance opportunities for musicians, ambassador programs and more. The content for The Institute has been designed and developed by Indigenous young people since 2005 and each year is enhanced and improved thanks to the input from our mentees, mentors staff and other stakeholders. Trained Indigenous facilitators who are supported by volunteer university student mentors, other AIME staff and a variety of special guests undertake the delivery of the Institute modules.

\section{Tutor Squads}

The Tutor Squad program features trained university mentors who head out to local schools and community centres to provide free academic support for Indigenous high school students. By providing additional tutors outside of the AIME Institute, we are aiming to provide opportunities for students to not only build the confidence and self-esteem required to make it through school but also the literacy and numeracy skills required to do so. Through the Tutor Squads, mentors help with personal learning plans, study plans, goal setting, homework, assignments and any other work the students may need assistance with. We have also created AIME Radio, which is a podcast featuring educators, mentors, mentees and others in the field to help tutors learn more about the power of education.

\section{One-on-one coaching, career support and post-school transition}

AIME endeavours to get to know the students throughout their high schooling experience, so that when it comes to their senior years we can provide the best possible advice, support and targeted opportunities for each student to be able to transition into university, further education and training or employment once they complete Year 12. During the course of each year, AIME works with its corporate and university partners, to source post-school opportunities for their mentees. AIME then follows up with each student to ensure they have the best possible chance of succeeding in their first foray into the world beyond high school.

AIME continues to stay formally connected to each mentee and provide mentoring support for the first six months of their university course, training or employment. AIME connects the mentees who progress into university with Career Trackers and encourages them to become AIME mentors. On the employment side of things, AIME has partnerships with some of Australia's biggest employers who are committed to increasing Indigenous employment. AIME works with these partners to ensure that AIME mentees are given the best chance to succeed as they enter the workforce. AIME also work with the students to help them transition into any other further education or training program they are interested in.

\section{Growth of the Program}

Since commencing in 2005, AIME has experienced exponential growth and great success. From 25 mentors and 25 mentees at one university site in 2005, the program has grown to 
have more than 4300 participating mentees and 1500 university student mentors in 2015. The program is currently delivered at 18 Australian universities across 35 different campuses with 317 participating schools in Queensland, New South Wales, Victoria, The Australian Capital Territory, South Australia and Western Australia (Fig. 1).

Figure 1: AIME sites 2015

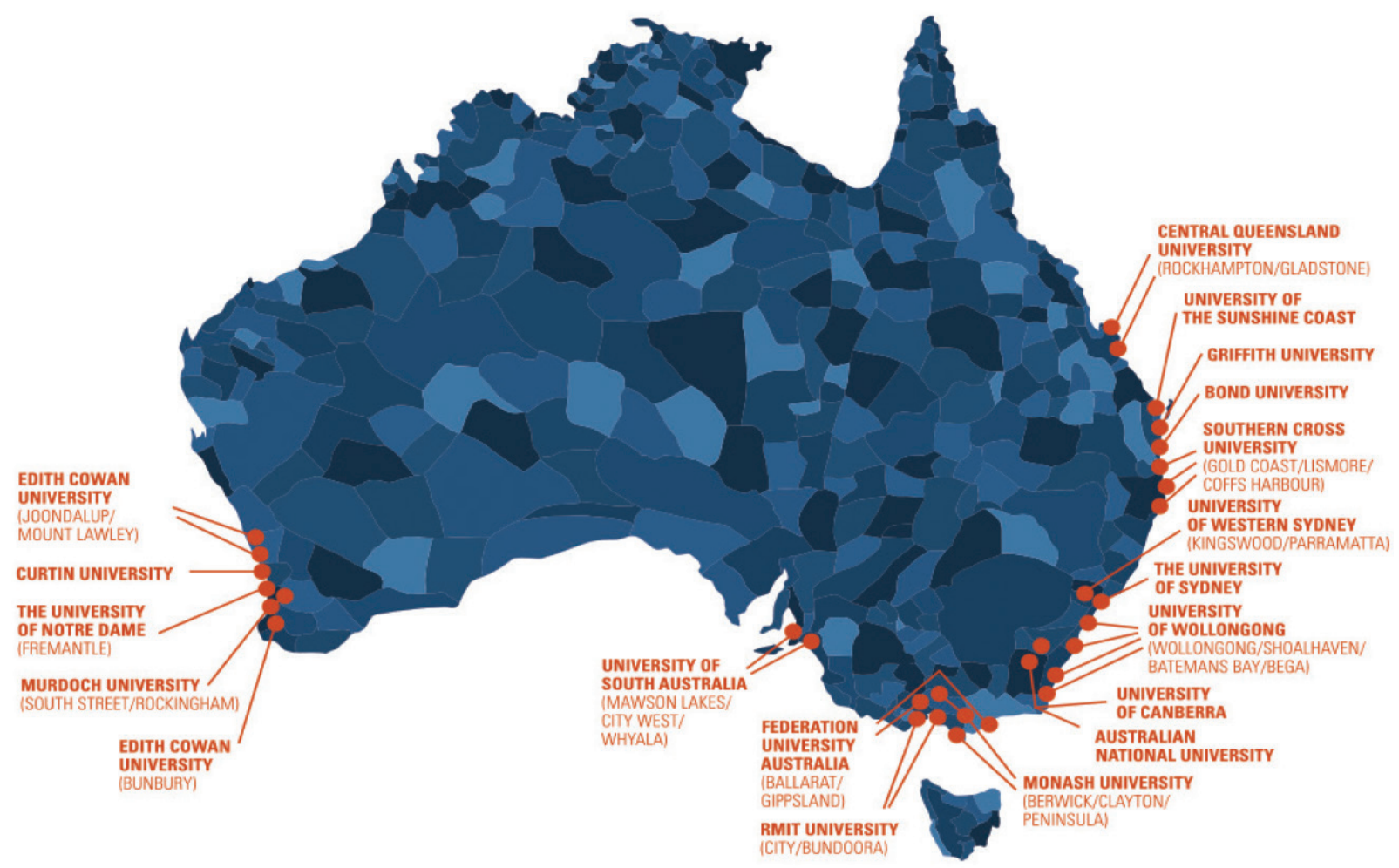

\section{Impact and results}

AIME believes that every single student's life and educational journey counts, so this is measured, recorded and reported on accordingly. Each year, AIME not only works with the students, but also with partner schools and research partners so AIME can measure the outcomes of the program in the most accurate and comprehensive way possible. The impact of the program is measured year on year, with statistics published in the AIME Annual Reports. Over the last six years, AIME students have consistently achieved school progression (Fig. 2), Year 12 completion and university admission rates significantly higher than national Indigenous statistics (AIME, 2014). Due to this, AIME has been cited as one of the most comprehensive and successful mentoring programs to engage Indigenous high school students (Lester \& Munns 2011; Bodkin-Andrews et al. 2013, SCRGSP, 2014). 
48

Figure 2. AIME'S outcomes, 2014
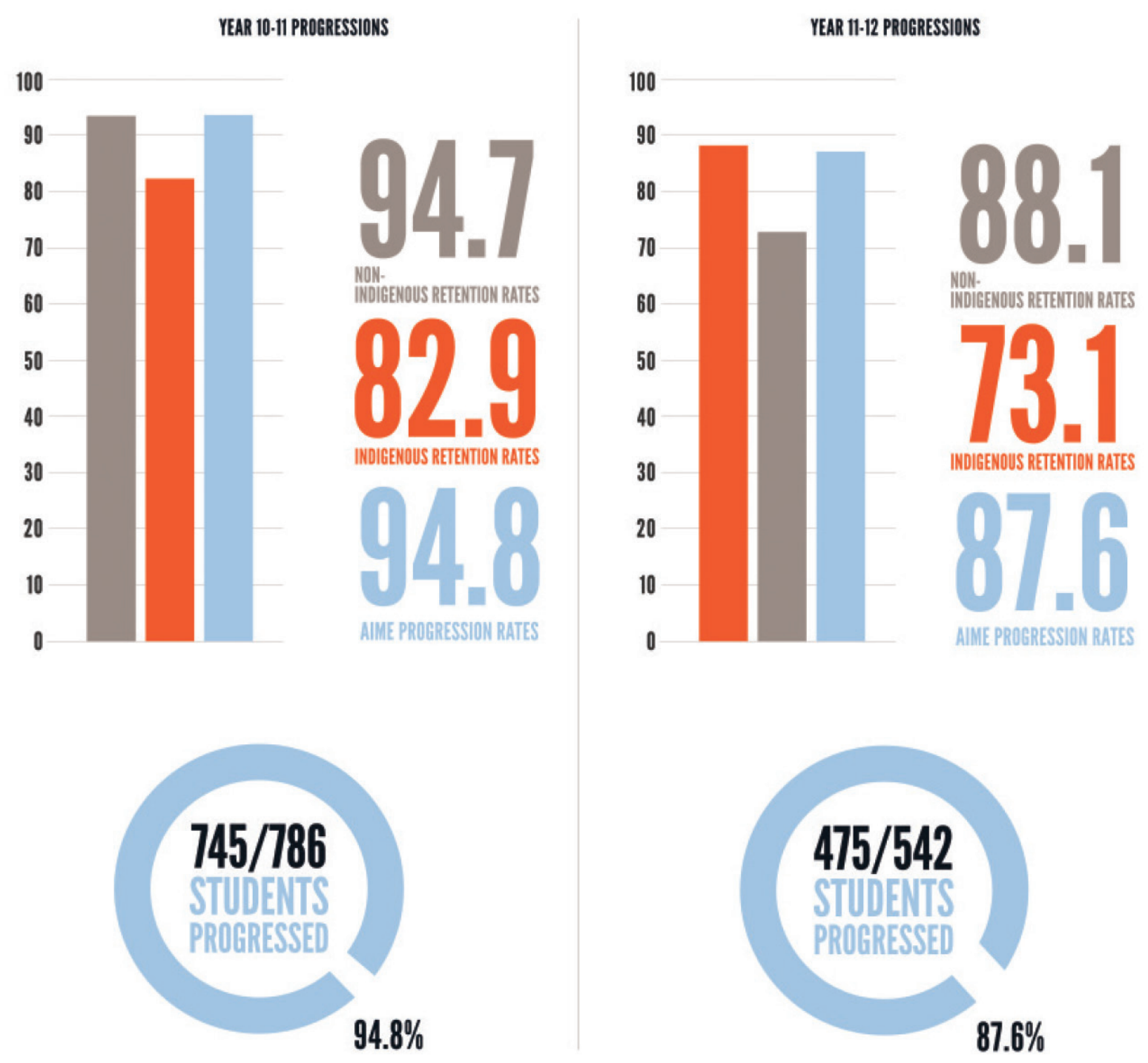

\section{YEAR 12 ATTAINMENT RATE}
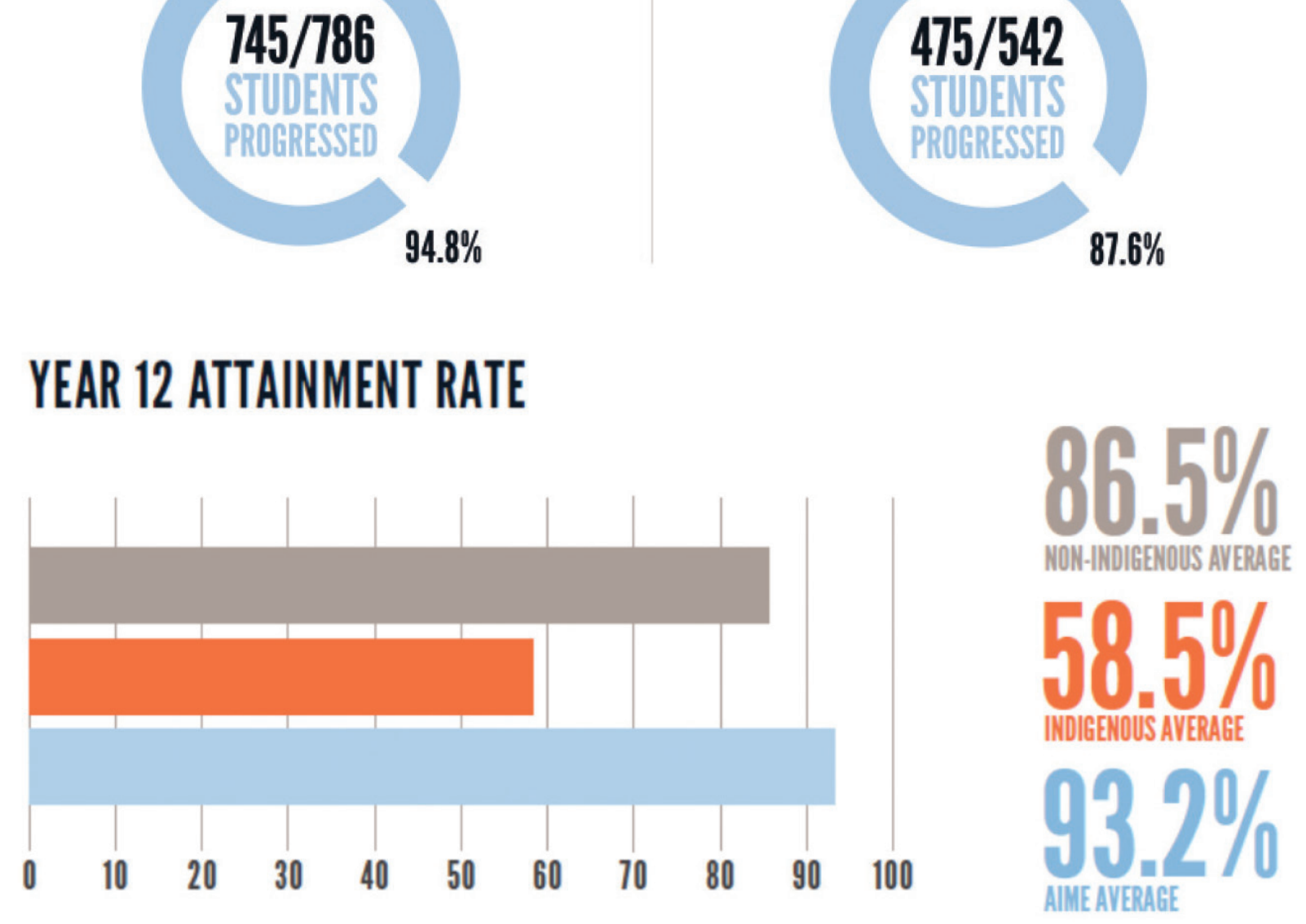
One of the most pleasing results from 2014 was that $93.2 \%$ of the AIME Year 12 cohort completed Year 12. These students are leading the way on meeting the COAG target of halving the gap in Year 12 attainment, by surpassing the national non-Indigenous rate by 6.7 percentage points and the national Indigenous rate by 34.7 percentage points (AIME, 2014; SCRGSP, 2014). AIME students are also leading the way in closing the gap on post-school pathways into university, further education and training and employment with nearly a third of this group (30.9\%) successfully transitioning onto a university pathway.

\section{Independent research}

In order to better understand the impact of the program and the possible long-term effects on the participants, AIME has commissioned a number of key independent research projects. The first was a large-scale independent evaluation into the effectiveness of the program, scalability and the associated mentee outcomes (Harwood et al. 2013). Findings indicated that AIME reached its objective of encouraging better school grade progression rates for Indigenous students, compared with the national average and that AIME positively impacted:

- The strength and resilience of mentees;

- Mentee pride in being Indigenous;

- Mentees making strong connections with Indigenous peers, role models and culture;

- Aspirations and engagement for finishing school;

- Aspirations for continuing to further study;

- School retention rates.

Building on this research, a quantitative study by Bodkin-Andrews \& Harwood et al., (2013) found that Indigenous students participating in AIME:

- Were significantly more likely to aspire to complete high school (1.87 times more);

- Were significantly more likely to aspire to and attend university (1.30 times more);

- Had a higher sense of school self-concept;

- Had a higher sense of school enjoyment.

The strengths of the program were also backed up by an independent economic evaluation conducted by KPMG. Findings from this study indicated:

- $\quad$ AIME students performed better than Indigenous students around the country, and reached levels of school performance close to their non-Indigenous peers;

- Due to the mentoring benefits for students, AIME students are likely to be more employable and earn more;

- $\quad$ An AIME student that completes a university degree can be expected to earn up to $\$ 332,000$ more over their lifetime compared to an Indigenous student that does not complete high school;

- $\quad$ The AIME program generates $\$ 7$ in benefits for every $\$ 1$ of cost (KPMG 2013).

\section{Three key ways AIME educates and engages its students}

Three of the key ways that AIME educates and engages its students have been identified through analysis of current and completed research: (1) the power of storytelling; (2) aligning positive associations with identity to give the students another source of pride and strength; and (3) creating a fun, safe, welcoming yet challenging environment to help the students flourish. 


\section{The Power of Storytelling}

AIME believes that every word is precious, every sentence written or uttered is a chance to change a life, so there is a relentless chase for perfection in communication. Throughout the ancient cultures of the world stories have been used to pass on information about morality, lifestyle, law, and survival. Today, the AIME program is inspired by the previous generations of Aboriginal and Torres Strait Islander people that have transferred information between generations through stories, and by some of the great communicators that have inspired and impacted the evolution of thought through the Western World, like Shakespeare, Plato, Aristotle and Socrates.

Stories are used to captivate, inspire and unlock the students' imagination. Every one of our 49 modules is intentionally scripted like a play, so students are engaged from the commencement of each session all the way through to the end. Instead of providing facts and figures and using an authoritative style of teaching, stories and storytelling is used to paint scenarios and teach ethical lessons. The use of storytelling is also engineered to provide students the opportunity to take ownership and leadership over their educational journey. A high expectations learning environment is created where students are challenged to step out of their comfort zone, engage in school and believe that they can achieve anything.

"From this moment, you have a chance to reach for worlds unimagined, to dream of futures made in movies, and then to make the commitment to work harder than any other kid your age, harder than anyone that has come before you, harder than anyone who will come after you, in order to bring your dreams to life. Because that's what it takes". (AIME 2014).

This high expectation, inspirational narrative is interwoven throughout all of the sessions forever encouraging the students to reach their goals.

When building relationships and rapport with the students, AIME staff use personal stories to help relate the main messages to the students lives. It has been found that students take the messages a lot more seriously when staff are honest, vulnerable and share real life experiences. By sharing stories with the students and not telling them what is right and wrong, the program is able to navigate, explore and discuss decisions and choices that are available to them, empowering them to make the right decisions.

Although the session content remains the same across the country, stories are tailored to the different communities the sessions are being run in. Local staff are employed at different sites that know and are from the local community and local people are invited to share, relate and personalise the stories. Students are also sometimes from different communities so they also have the opportunity to share stories with their peers so there is constant learning, teaching and sharing stories from other communities. This in turn provides the ability to create relationships through stories and connections between different communities.

\section{Aligning positive associations with identity}

Specific Identity sessions are held in the Year 9 and 10 programs with the objective of establishing the framework that Indigenous = Success and that identity can be a source of strength. The simple message is that to be Indigenous is to hold a position of strength, not a position of weakness. Sessions are all-inclusive and a safe space for students who speak Aboriginal languages as well as for students who don't know anything about their heritage or feel disconnected. Some students may want to know more about their culture and identity but don't know where to start. Some students may feel that hey don't belong, either getting 
challenged on where they are from and not knowing, or getting challenged on their skin colour. Learning about identity though is interwoven throughout the whole program. Through stories and discussions, students learn more about their identities and they are encouraged to continue to learn and discover these outside of the program.

AIME presenters are all Indigenous role models and are proud, strong, successful leaders. They get in front of the students showcasing Indigenous success. Through their personal testimonies, they describe their journeys to where they are today, cementing the fact that being Indigenous is not an excuse for failure, it is a reason for success. Staff describe what it means to them to be Indigenous, and that there is no right or wrong answer. All of our staff have different skin colours, different levels of connection to their Indigenous storylines and culture and different engagement with Indigenous people every day. Some live in communities and some don't. This honesty and vulnerability to share their personal stories creates a safe space for the students to explore, discuss and learn about their own identities.

Another way Indigenous success and different identities is showcased is through inviting Indigenous guests to the program to share their stories with the students, including the pathways they traversed to get to where they are today. The philosophy is to try and change mind-sets. Some students know their culture and are strong in their identity but haven't seen a range of Indigenous people with successful and varied careers.

Discussing and exploring positive identities has been a great tool in tackling shame which, for some students, can be related to identity On the first day, all students sign a contract agreeing to a number of rules and high expectations. No Shame at AIME is one of these important rules and is reiterated constantly through out the program.

\section{Creating a fun, safe, welcoming and challenging environment}

AIME endeavours to unlock the magic of learning and help be a vessel that can change young people's views of learning forever. To do this, there is an emphasis on making the learning environment fun, safe, welcoming and challenging.

Since AIME commenced in 2005 , the program has been continually refined to stay relevant, fun and engaging. This relates not only to content evaluation and enhancement, but also to the vibe that has been purposively create. Each moment with students is potentially a life changing opportunities so a vibe and environment is created that will allow these 'moments' to occur. Each AIME day is likened to a show, where no detail is left to spare in order to ensure the most immersive learning experience possible. The performers are well rehearsed; locations are carefully selected; resources are prepped and ready; each learning module is scripted with timings worked out to a tee; the lighting, audio-visuals and room set up are carefully planned; specific music is selected and played throughout the day; and all of the staff and mentors are prepped and ready to go, wearing their AIME hoodies. The vibe and learning opportunities commence as soon as the students arrive on the university campus. Some students have been to a university campus before, yet for some, an AIME day can be their first experience so it is important to showcase the university and what it can offer, but to also make each and every experience unforgettable.

The AIME vibe is created through a relaxed yet supportive atmosphere but it is also a high expectations environment. The philosophy is to try and make the students feel comfortable and safe enough to step out of their comfort zones, participate in all activities, increase their confidence and have the ability to then do the same when they get back to school. That is, have increased confidence to ask questions in class, participate in public speaking, stand up for leadership opportunities and similar attitudinal and behavioural attributes. 
Through this supportive, high expectations environment, deep relationships are built with the students. Through these relationships, the students feel comfortable discussing their goals and AIME staff are able to help provide tailored support throughout school and then one-onone post school career coaching.

Research into the program describes how AIME conceptualises and promotes the program as 'meeting a deficit of the educational system, as opposed to a deficit of the young person' (Harwood et al. 2015). Instead of concentrating on raising each student's aspirations, AIME places a focus on reversing the low expectations and lack of support that Indigenous young people may encounter in school settings by creating a high expectations environment and vibe.

\section{Positive post-school pathways}

Progressing through high school, completing Year 12 and successfully transitioning to post school pathways can be an extremely complex and daunting process. In order to help students navigate and traverse high school with the best possible outcomes, these issues are addressed throughout the program. Through storytelling, aligning positive associations with identity and creating specific learning environments, there have been greater levels of success evidenced by high-grade progressions and Year 12 attainment rates. The next step in the process is helping mentees traverse the post school transition stage.

Post school transition support is one of the three pillars of the AIME program. From Year 7 all the way through to Year 12, students participate in the program at local university campuses building on their cultural capital of universities through time on campus, interaction with university staff, Indigenous centres and their university student mentors. There are a number of ways that post school transition support is provided. Staff work with the students both in sessions and on school visits to develop individual plans for their future. An example is the AIME Yearbook Portal which is an online application that connects Year 12 AIME students with education and employment opportunities being offered by AIME's university and corporate partners. The students and staff work together to establish individual online profiles and then AIME staff continue to work with the students providing whatever transition support is needed.

The skills and confidence the students build, the content they learn, the vibe and environment they receive and the relationships built throughout the program are what makes the transition process so much easier and why we believe we our students are achieving such great results. AIME has walked with the students from Year 7 to 12, continually challenging them to work harder, dream bigger and to step out of their comfort zones. The 2014 AIME Year 12 cohort went better than the national average for non-Indigenous 17-24 year olds engaged in post school education, training or employment (76\% compared with 75\%) (AIME 2014 \& SCRGSP 2014). AIME has put even more resources towards the 2015 Year 12 cohort in the belief that even better results are not only possible but are achievable. 


\section{References}

AIME. (2014). 2014 Annual Report. Retrieved from http://www.aimementoring.com/about/reports/.

Bodkin-Andrews, G., Harwood, V., McMahon, S. \& Priestly, A. (2013). AIM(E) for Completing School and University: Analysing the Strength of the Australian Indigenous Mentoring Experience. In R. Craven \& J. Mooney (Eds), Diversity in Higher Education: Seeding Success in Indigenous Australian Higher Education, Volume 14 (pp. 113-134). Bradford, UK: Emerald Group Publishing.

Commonwealth of Australia Department of Industry, (2014). Statistics publications.

Student 2013 full year: Selected higher education statistics publication. Retrieved from the Department of Education and Training website: https://education.gov.au/ selected-higher-education-statistics-2013-student-data.

Harwood, V., Bodkin-Andrews, G., Clapham, K., O’Shea, S., Wright, J., Kervin, L., \& McMahon, S. (2013). Evaluation of the AIME Outreach Program. Wollongong, Australia: University of Wollongong.

Harwood, V., McMahon, S., O'Shea, S., Bodkin-Andrews, G., \& Priestly, A. (2015). Recognising Aspiration: The AIME program's effectiveness in inspiring Indigenous young people's participation in schooling and opportunities for further education and employment. The Australian Educational Researcher, 21(2), 217-236.

Kervin, L., McMahon, S., O'Shea, S. \& Harwood, V. (2014). Digital storytelling: Capturing the stories of mentors in Australian Indigenous Mentoring Experience. United Kingdom: Sage Publications.

Lester, J. \& Munns, G. (2011). Closing the Gap. In R. G. Craven (Ed.), Teaching Aboriginal Studies (pp.229-256). Sydney, NSW: Allen \& Unwin. Retrieved from https://education.gov.au/ selected-higher-education-statistics-2013-student-data.

KPMG. (2013). Economic evaluation of the Australian Indigenous Mentoring Experience program: Final report. Retrieved from http://www.aimementoring.com.

O'Shea, S., Harwood, V., Kervin, L. \& Humphry, N. (2013). Connection, challenge, and change: the narratives of university students mentoring young Indigenous Australians. Mentoring and Tutoring, 21(4), 392-411.

O' Shea, S., Chandler, P., Harwood, V., McMahon, S., Priestly, A. \& Bodkin-Andrews, G. (2014). Working together to increase Indigenous students' educational participation nationally: A case study of partnership between the Australian Indigenous Mentoring Experience (AIME) and the University of Wollongong. In the National Centre for Student Equity in Higher Education (Ed.), Partnerships in Education. Perth.

SCRGSP (Steering Committee for the Review of Government Service Provision). (2014). Overcoming Indigenous Disadvantage: Key Indicators 2014. Canberra: Productivity Commission. 